\title{
Identifying Variable
}

National Cancer Institute

\section{Source}

National Cancer Institute. Identifying Variable. NCI Thesaurus. Code C83293.

The variable that identifies a particular data element. 